\title{
Inhnaltsiiller ridgt.
}

:Lliiryungent . . . . . . . . . . . . . . . . XIV

Et bünsungen und beriftipungen . . . . . . . XV

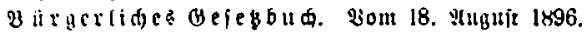

vit is

Eriter vbidnitt. - Herionen.

Eriter Titel. - Matiurtide Herfonet. \$\$ 1-2), 1

Buveiter Titel. - Jurifilf berionen.

I. Vereitte.

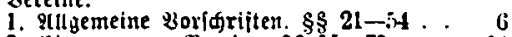

2. Cingetragene Vereine. $\$ \$ 55-79$. . . 14

11. Ftijtungen. $\$ \$ 80-88 \ldots . . . . . .18$

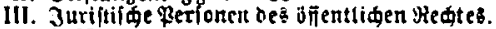
$\$ 89$................. 20

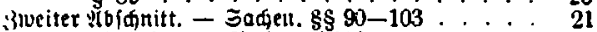

Tritter Ploidnitt. - Medtsgeidufte.

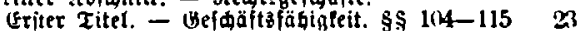

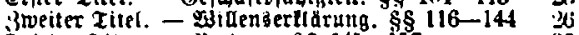

Eritter Iitel. - Vettrag. \$8 145-i57... . 33

Lierter Iitel. - Bebingung. - Zeitbejtimmung.

s\$ $158-163 . \ldots . \ldots 35$

तibifter Titel. - Bertrctinas. - Eollatad.

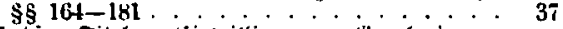

Eediter ritel. - Einidligung. - Genebmigung.

SS $18 z-185$. . . . . . . . * * . 41

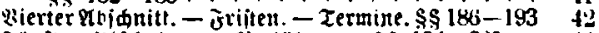

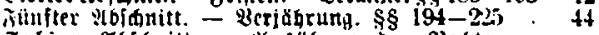

Eediter Rofanitt. - Yusubung oer redte.

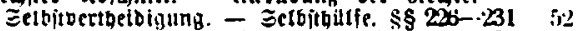

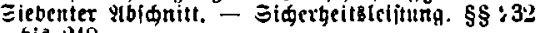

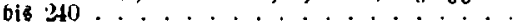

3ucites gud. - Hedt oer Бdulobergät= niffe.

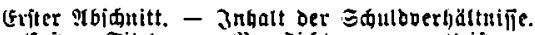
Eriter Titel. - Berpilidtuth jur Leiptung. S\$ $241-292$. . . . . . . . . . . . . 16 Brveiter Titel. - Berjug des siäubigers. \$\$ 293-304. . . * . . . . . .

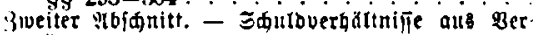
tringert.

Erftex Titel. - Begrintung. - 3nhalt des Bertrags. \$S 305-319........... 3ivetter Iitei. - Begenleitiger Lertrag. \$\$ \$g)

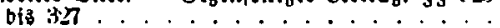
Eritter Titer. - Rerforeden ber Leiitung an cinen Dritten. $\$ \$ 328-335$. . . . . . . . . Bierter Titel. - Traufgabe. - Bertragsitrafe. s\$ $336-345$. . . . . . . . . 76 Finfter Titcl. - Mildtritt. $\$ \$ 346-361^{\circ} \cdot \dot{*}^{\circ} \cdot$ is $^{\circ}$ 


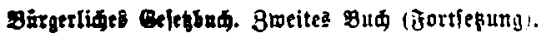

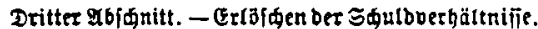
Griter Titel. - Erfilaung. $\$ \$ 362-371 . .81$

3weiter Zitel. - Sinterlegur. \$\$ 372-386. 81

Drttter TiteI. - शtufrednung. $\$ \$ 387-396, \quad 86$

Bierter Titel. - Grlof. \$ 397 . . . . . 8

Bierter Yb/dinitt. - liebcttragung ber forberuit]. $\$ \$ 398-413 . . . . . . .8 \%$

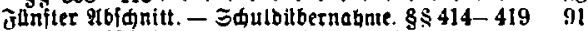

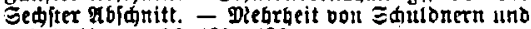
Gläubigern. \$\$ 420-432.

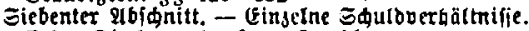
Eriter Titel. - sauf. - Taul由.

I. qugemeine Sorimriften. \&8 433-4ix . . si

11. Getoührteifung negen plängel der 三ode. $\$ \$ 459-493$.

III. Uefonbere Wrten bes staffes.

1. Srauf nad Probe. stalif auf Brobe. $\$ \$ 494-496$. . . 107

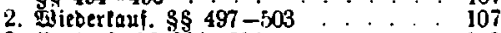

3 Borlauf. $\$ \$ 504-514 . \ldots 111$

IV. Tauid. \& $515 \ldots \ldots$

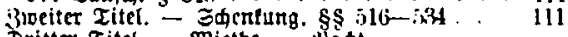

Iritter Titel. - Pliethe. - waht.

1. Wiicthe. $\$ \$ \$ 35-50011 \mathrm{1}$

11. Wa由t. $88581-597$ 1.5

Wierter Zitet. - Leibe. $\$ \$ 590-606 \quad . \quad 1: 9$

Fitnfter Titel. -. Tarleben. \$\$ 60i-610 . . 130

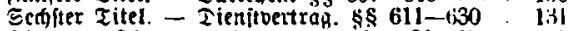

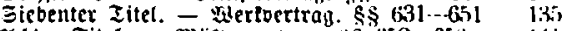

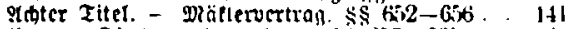

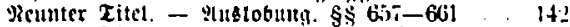

3chnter Titel - onftrag. \$s $612-676$. 14i

Eliter Iitel - Geidüftsilbrutg obte suitra!n.

$\$ \$ 677-68 i \ldots . . . . .14 i$

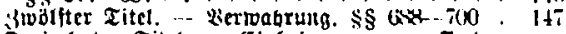

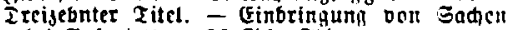

bei (5aptwirtbert. \$\$ 701-704. …. 149

Hieraehnter Titel. - Weiedidaft $\$ \$ 705-740: 150$

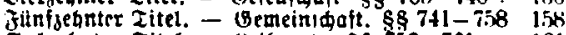

Sedsehnter zitel. - Leibrente. \$\$ $759-761.161$

Elebjebnter Titel. - Epiel. - gistte. $\$ \$ 762-i 6416^{\circ}$

qutjebnter Titel. - Uitrgidaft. \$\$ $76 ;-778,162$

Reutzebrter zitel. - Bergleid. \$ 779 .... 166

Blwandigiter Titel. - Equloveripreden. -

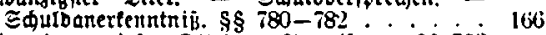

ctrumbsrounsigfter Titc!. - Imbsifung. \$s 78; -792 . 167

3roelundzwanglgiter Titel. - ₹dultoveridrcibuty auf ben 3nhaber. $\$ \$ 793-803 \ldots \ldots$

Treiunb3wantigiter Zitcl. - Zorlesunt bon

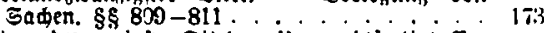

Bierunbjivanjigfter Titel. - llngeredtiertigte $B_{c}$ reiderung. $\$ \$ 812-822 \ldots . . . .174$

Gunfutnosmanjigfter Titel. - interlaubte jano.

lungen. $\$ \$ 823-85.5 . \ldots . . . .176$

Drittes Bud. - Sa中enredt.

Exfter وbínitt. - Befip. \$\$ 854-872 .... 185

8 weiter gbidnitt. - gugemeine goridiften ilber

Rewte an Grundftiden. $\$ 8873-902 \ldots 188$ 


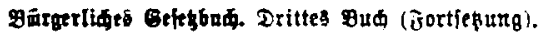

Tritter qGidnitt. - Eigentádm.

Griter Titel. - 3rbalt bes Eigenthume. \$\$ 903 bis 924

Sweiter Titel. - Enverb unt Berluit bes Etgenthum all ortunbitutten. $\$ \$ 925-928 \ldots$

Erliter Ittel. -- Grtuetb utmo werluit bes figen thlums an beweplinen saden.

1. Llebertraburg. $\$ \$ 929-930 \ldots . . .204$

Ii. Eriifung. \$s $937-945$. . . . . . . .

III. Kerbindunt. - Jermijinng. - Berar: beituth. $\leqslant 546-052 \ldots 20 \%$

IV. Ermerb bon Erjeugnifien und lonitigeit Be: irastbtbeilen ciner $\Leftarrow$ cde. $\$ \$ 93-05 \%, 209$

V. 9tnei!nung \$\$ 958-9u4. . . . . 210

v1. Fund. $\$ 8965-964$. . . . . . . 211

Wierter Zitel - Unjpritde aus bem Eigenthum. $\$ \$ 950-1007 \ldots . . .215$

Filnfter Titcl. - Vitetgenthum. s\$ 100k-1011. 2\$1 Wierter noidnitt. - Erobauredt. \$\$ 1012-1017. 221 jünfter grbidinitt. - Eienjtbateiten.

Eriter Zitel. - Brunboiemitbatfeiter. $\S \S 1018$ biz $10 \% 9$.

3weiter Titel. - Tiésgraud.

I. grię̧braud) an Saden. sș 1030-1067. . 244

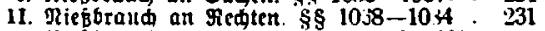

11I. Riésbraud an ciltcm Bermigen. \$\$ 1085 bita 1039 .

Iritler ZiteI. - Bejめräntte periönlide Zienjt. variciten \& $1090-1093$

Ecdjiter צbfanitt. - Bortaufbredt. \$\$ 1094-1104.

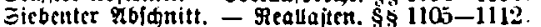

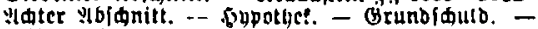
Reittenídulo.

Erjler Zitrl. - Soporbet. \$\$ 1113-1190 ...

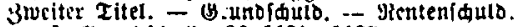

I (irundi由ulo. \$s 1191-1198.

II. Tenteríduld \& $1199-1203$

Vicunter Fbifitt. - Bjanoredt an berwegliden כaden und alt gichten.

Erfter Titel. - Pfandreds an bejoeglihelt saden. \$\$ 1204-1272. . . . . . म

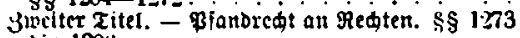
bis 1296.

Bicrtes 8 ud. - jantilienredt.

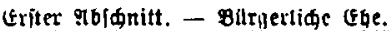

Eriter Titel. - Berlöbni Guveiter Titel. - Eingehung ber ffle. \$\$ 1303 bis $13: 22$

Iritter Titet. - Nidtigfeit uno vinfedtbartent bex Ëbe. $\$ 81323-1347$. . . . . . . .

Wietter Titel. - wicherwerbciratbust in jalle Der Todeserllarun 8 . $\$$ 1348-1352 . . . . .

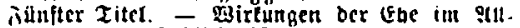
gemcinen. $8 \$ 1353-1352 \ldots$ Sedifter Titei. - Eqelides Bitterredt.

I. Befezlides biterredt.

1. भunpemeine Boridrifter. $\$ \S 1363$ big 1372 


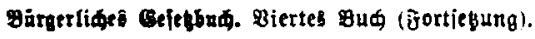

2. Berwaltung unb gtubniegung. $\$ \$ 13: 3$ bie $1409 \ldots . . .295$

3. Squbertaitung. $\$ \$ 1410-1417 \ldots$

4. Deenbigung Der Berroltung und $\Re$ ht: niefurg. \$3 1418-1+25.... 304

5. Bittertrennun 8 . 88 1428-1431 . . . 30

II. Vertragemásiges oütertedit.

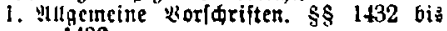
$1436 \ldots$

2. Mugemeine (Biltergeneirigaft. $\$ \$ 1437$ bi 1518 . 300

3. Errurgenimuit Bemecinimait. $\$ \$ 1519$ bis $1548 \ldots . . . . .324$

4. ifabraibgemeinidaj. \$s $1549-15 i 7$.

III. Bitterredtercgitter. $\$ \$ 138-1563$. . 3 3it Ziebenter Titet. - Eheibung ber che. \$\$ 1564 bis $1587 \ldots \ldots \ldots$

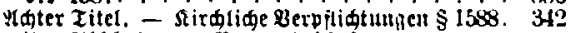

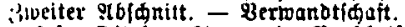

viriter Titel. - :ugemeine $B$ orfdriften. $\$ \$ 1589$ bis 1 is $90 . . . . . .342$ imeiter Titel. - Ebelide ribitamminit. \$s lish bi.? 1600

Iritter Titel. - L luterhaltspilidt. S\$ 1601-1 hils.

wiertel TiteI. - Redtlide כtellung Der chetidjest stintoer.

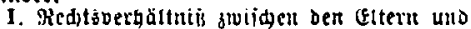
dem fimbe im Magemeinent. $\leqslant \$ 1611 ;$ bis 1625 . . . 34

11. Elterlite (Selvalt. $16 \%$. . . Biv

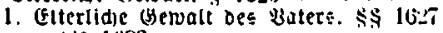
bis $1683 \ldots \ldots$. . . . . . .

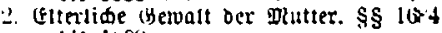
bis ll, ....................

iiinfter Fittl. -. Hedtlide Etelluny oer stisther

alts tidtigen (Eben. $3 \$ 1699-1704 . .$. .

Eediter Fitet. - Reditlide Etellung oer im-

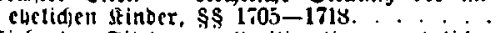

Sietentir zitel. - Eegitimation unetelider Minder.

1. Legitination burd namfolgende fibe.

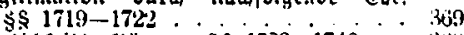

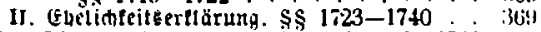

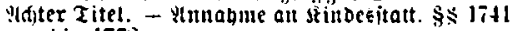
bis $17 \% 2$

Iritter voidnitt. - Formtindidaft.

Erriter Zitel. - Wormumbltalit über IRinier. jübrige.

1. Yinorinung ber Vornumbiquft. $\$ \$ 1773$ bis 1742........... 3T

1I. vithrung ber Bormundidait. \$s 1793 bis 18360.033

III. Fitriorge und 9cufridt ocs 3ormunb: imafisgeridta. \&s 1837-1848 .... 394

IV. Wiitwirlung bes Gemeinderbatfentatbs. \$\$ 1849-1851 397

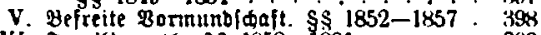

VI. Jomiltenrath. \&\$ 1858-1881... : 399

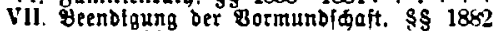
ถ⿻?口⺕ 1895 


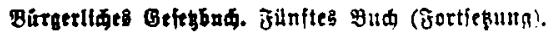

3weiter Titer. - Bormundidaft iber Boll-

iährige. $\$ \$ 1890^{\prime}-1908 \ldots \ldots 00$

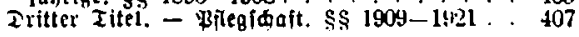

jitiftes

Eriter qbidnitt. - Erbfolge. 8s 1922-1941. . 410

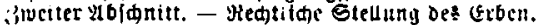

Erfter Titel. -- Tnnahme unt :Iuzi由lagung ber

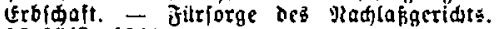

$\$ \S 1942-1060$. . . . . . .

310 eiter Titer. Daitung bes crben fikr bie

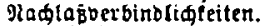

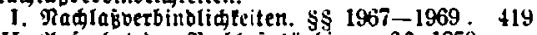

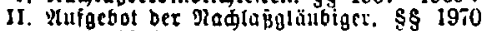
bil $1974 \ldots . . . . .419$

III. Beforïnlung ber syafturg bes Erben. $\$ \$ 1975-1992$. . . 421

IV. Bnventarerribtung. - unbeidränite jaj: tung bes Grben. \$\$ $1993-2013$.. 425

v. 2tufíieberbe Eimreben. $\$ \$ 2014-2017:+20$

Eritter Zitel. - Grbldaftenanjpru. \$\$ 2018

bis 2031 . . . . . . .

bierter Zitel. - Wiehrbeit bon (r ben.

1. Redtsoctbälmis ber frben uster einander. $\$ \$ 2032-2057$. . . 433

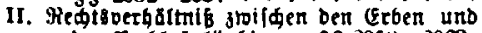

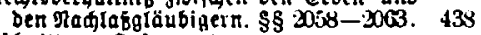

Iritter 2 bidnttt. - Zejtament.

Eriter ₹itel. - Magemeine Korjdriften. \$\$2064 bit $2086 . \cdots$.

Sineiter Titel. - Erbeinieguig $\$ 3087^{\circ}-2099$.

Iritter Tttel. - Giniesurg eittes Ramerben.
$\$ 82100-2146$. . .

Bietter Titcl. - Bermádnik. s\$ 2147-2igi.

ziluiter Titel. - quplage. \$\$ $2192-2196$.

כediter Titel. - Telamentisvodjtreder. \$§ $219 i$ bis 2238 . . . . tho

Siebenter Titel. - Erriatunt uno qufqebuti eines Teilaments. \$\$ $229-2264$. . . . .

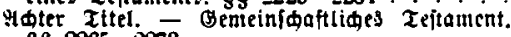
$\$ \$ 2265-2273$. . . . 480

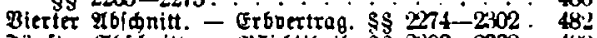

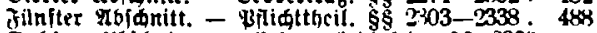

כeditct 16 /

bis 2345 . . . . .

Eiebenter gbidjnitt. - Frbbersidt. \$s $2346-2352.493$

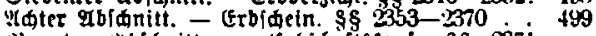

Hieunter ybidnitt. - Exhidaftetanf. \$s 2371

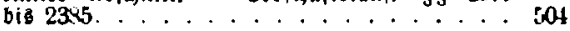

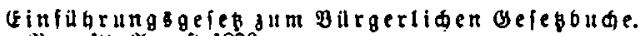
Bom 18. Tlubuft 1896.

Eriter Foldnitt. - TUyemeine Boridrijten. Itot. 1-31

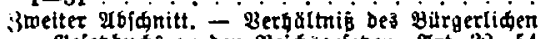

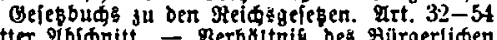

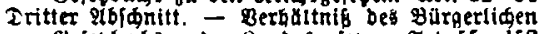

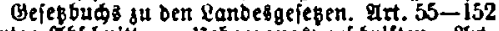

Bierter Rof $153-218 \ldots \ldots . . . .53$ 
Geics, betrefient die Tus äbrung bes gctlíen Gelebsums ta Elfab-lotgringen. Wom 17. Rpril 1899.

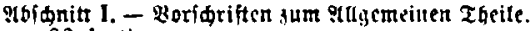
\$\$ $1-8$. .

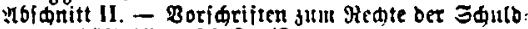
verbältmifie. $\$ \$ 9-\mathbf{4 3}$. . . . . . .

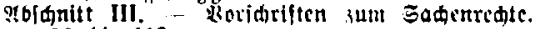
ss $4-116$

bejtummunen, vetrefrend bie Medie an Brunb itilden, fiir luethe dos Urrunbbut nod tid als anjelegt ausulehcu ijt. $\$ \$ 86-116$.

Ubidntt IV. - Boridriften ३un Jamilienredits. $S \$ 117-165$. .

Ilebergan refte. $\$ \$ 14-16 ; . . .2$.

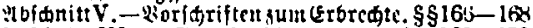

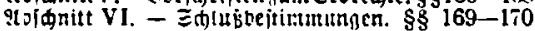

(Weieb il ber bit graplegengeltenderfreituil

liscul (Berimtebarleit. Nom 17. Diai 1893 .

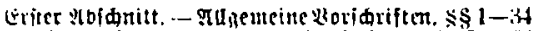

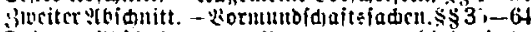

Triticr slbimititt. - YUtmabme an stinbceftatt. $5 \leqslant 65-64$

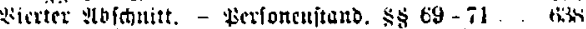

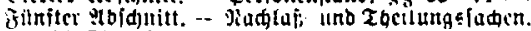
\&\$ $72-49$.

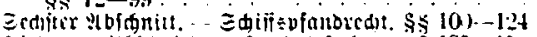

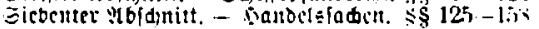

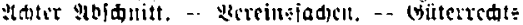
'eniitit. $\leqslant 51.00-10^{\circ}$.

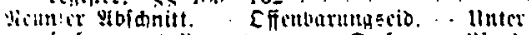

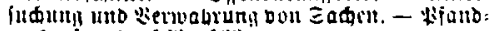
vertauf. $\leqslant \leqslant 163-160$

Bihuter gividititt. - (Herid)tlide und inotariclli lirfurtoct. $\$ \leqslant 167-184 \ldots$

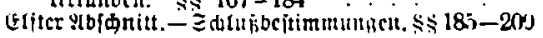

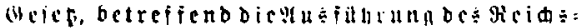
acfebes über bie :lngelegenteiten bet frei toilligett Beri申tsbartit. Wom 6. Noucmber 1899.

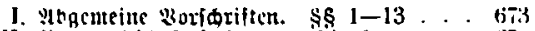

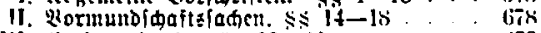

III, Werionetitand. $\$ \$ 19-2 ! \ldots \ldots$

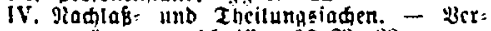

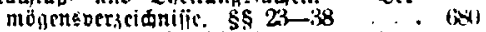

V. Dandelejaden.--Eonjtige stnaeleacnbeilcut.

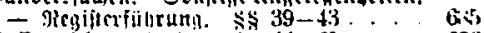

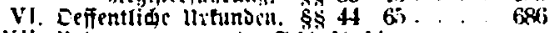

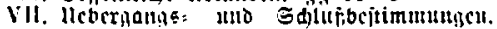

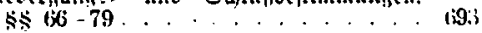

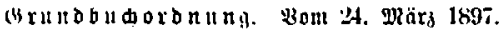

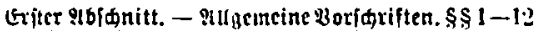
: Hoeiter Rbidnitt. - Eintrajungen indas Hrubbut. \$\$ $13-55$ * * - * - * - *

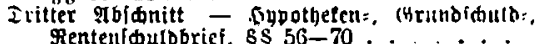




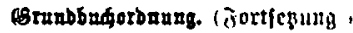

Bierter 9t6jqnitt. - Bejwucrbe. \$\$71-81 . . 712

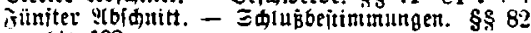
bis $102 \ldots \ldots \ldots \ldots . \ldots \ldots$

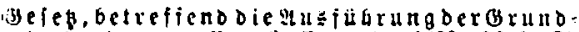
butartonuth. Hom 6. Nodeuber 1899. \$\$ 1-31 719

iscicb jiber bie 3toangeveriteigerung uno

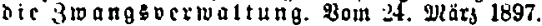

Eriter 2lbidnitt. - 3vangẼveriteigerung unb

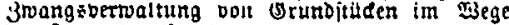
Det 3ivang soollitredung.

Eriter Titer. - MUgemeine Borfariften. \$§ 1 bis 14 .

Stweiter zitel. .- 3wangs periteigetung.

1. Inorbnung Der Betiteigetung. \&s 15-27. 730

II. Mufbebung und einitroeilige finjtellung bes Berfahrens, \&s $28-34$.....

III. Beitimmun bes velifeigerungstermins. s\$ $35-43$. . . . . . . . . .

1v. Beringites Bebot. - Beritciperuntgibe: bingungen. $\$ 544-65 . . . .736$

v. Serfieigerung. $66-78$. 743

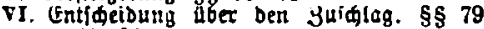
bis o4.

vII. Pef́́duerde. $\$ \$ 95-104$.

V11I. Betţetlung bes Crlöfes. \$\$ 105-145 . 752

Dritter Titel. - 3wargsverwoltung. $\$ \$ 1 \pm 6$

bis $161 \ldots . . . . . .$.

Simeiter Ubiqniti. - 3wangsoriteigerung von Shiffen tm wege der 3 jangegouptredung. \$\$ 162 bis 171

Dritter gbidnitt. - 3twang beriteigerung und 3mang bocrwaltung in belonberen Fallen. \$\$ 172 bis $184 \ldots . . . .$.

Einfubrungseleb su bem beleb thber bie Blvangs beriteigerung unb die $\mathcal{B}$ mang verwaltung. Zom 24. WRăr 1897 .

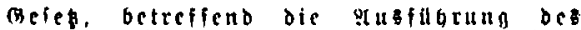
Heidseciebes itber bie 3 wangsocritei

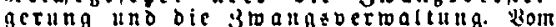
13. 9lobembe: 1:99.

Griter Mbidnitt. - Vollitredungsbeante. \&\$ $1-5$. 3tweiter Yofonitt. - 3wangsveritetgerung uno jroangevenoaltung tin wege ber 3rangsvoll: irredung. $\$ \$ 6-19$. . . . . . . .

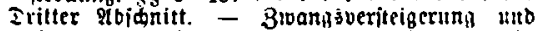
Groangeverivattung von gergwert eigenthunt in belonderen zăllen. $\$ \$ 20-25$. . . . is

Pierter qbidnitt. - llebergang : urb Edils. bejtimmuntaen. $\$ \$ 26 \rightarrow 77 . . . . . .$. 